Case Report

\title{
RECURRENT PYOGENIC GRAULOMA - A CASE REPORT
}

\author{
Amitha Ramesh ${ }^{1} \&$ Agumbe Priyanka Prakash ${ }^{2}$ \\ ${ }^{1}$ Professor, ${ }^{2}$ Post Graduate, Department of Periodontics, A.B. Shetty Memorial Institute of Dental Sciences, Nitte \\ University, Mangalore, Karnataka, India. \\ Correspondence : \\ Agumbe Priyanka Prakash \\ Post Graduate, Department of Periodontics. A B Shetty Memorial Institute of Dental Sciences \\ Nitte University, Mangalore - 575 018, Karnataka, India. \\ Mobile : +919449752055 E-mail : agumbe.priyanka@yahoo.co.in
}

\section{Abstract :}

A non-specific, conditioned gingival enlargement, pyogenic granuloma is a reactive lesion that can occur anywhere on mucosa or skin. In the oral cavity, the gingiva is the most frequent site. The recurrence rate is up to $15 \%$. The most common cause of pyogenic granuloma is local irritating factor. This case report describes a recurrent pyogenic granuloma in a 35 year old patient and its successful surgical management.

Keywords : pyogenic granuloma, recurrent, incisional biopsy.

\section{Case report:}

A 35 year old male patient reported to the department of periodontics, A B Shetty Memorial Institute of Dental, Sciences $M$ angalore with a chief complaint of swelling and discomfort in the inner side of lower anterior teeth since one month. The swelling which was gradual in progression, associated with pain and bleeding on trauma while eating or brushing. He gave a history of recurrence three times in the last one month at the same site. He gave history of a similar lesion 4 days back at the same site for which the patient had undergone surgical excision.

On clinical examination, a localised gingival swelling pink to red in colour measuring $1 \mathrm{~cm} \times 0.75 \mathrm{~cm}$ was present with respect to lingual aspect of left mandibular central incisor which was bleeding on probing that area. The growth was firm on palpation, non - tender with absence of discharge. Physical examination revealed no cervical lymphadenopathy or any other abnormality. On hard tissue examination there Access this article online Quick Response Code

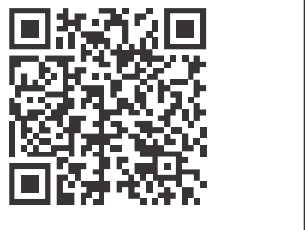
was no trauma form occlusion no mobility detected. There was moderate supragingival and sub gingival calculus present in that tooth. Patient presented no relevant medical history. The periapical radiograph showed no detectable bony defect. Based on the history, clinical and radiographic features a provisional diagnosis of pyogenic granuloma was made and the lesion was planned for excisional biopsy under local anesthesia.

First a thorough conventional non - surgical full mouth scaling and root planning was performed with curettes. There was moderate bleeding which could be controlled within a few minutes on applying pressure with gauze. After local anesthesia, with the help of a 15 no. BP blade the lesion was excised completely by trimming the remnants of soft tissue to prevent recurrence of the lesion. Antibiotics and analgesics were prescribed for 1 week. The excised tissue was then sent in formalin for histopathological examination.

Histopathological examination of $\mathrm{H}$ and $\mathrm{E}$ stained sections show epithelium and connective tissue. Epithelium is parakeratinized stratified squamous type. The connective tissue shows numerous blood vessels, chronic inflammatory cells like lymphocytes and plasma cells. Extravasated RBCs were also seen. The histopathological examination confirmed the clinical diagnosis of pyogenic granuloma. The patient was recalled after a week and the excised area was evaluated. Healing was satisfactory. 


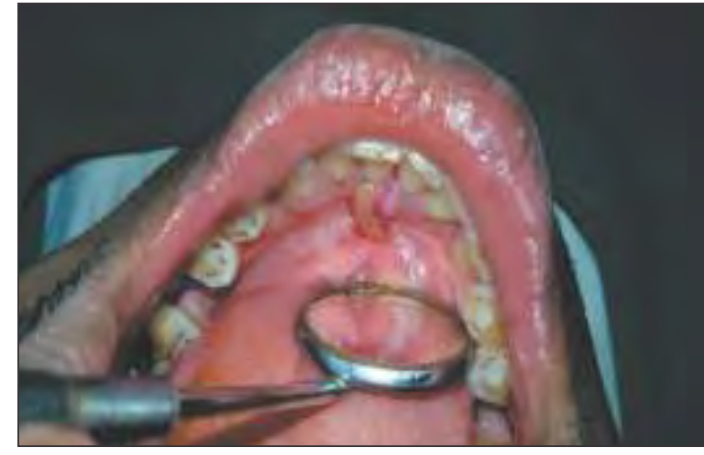

Pre-operative

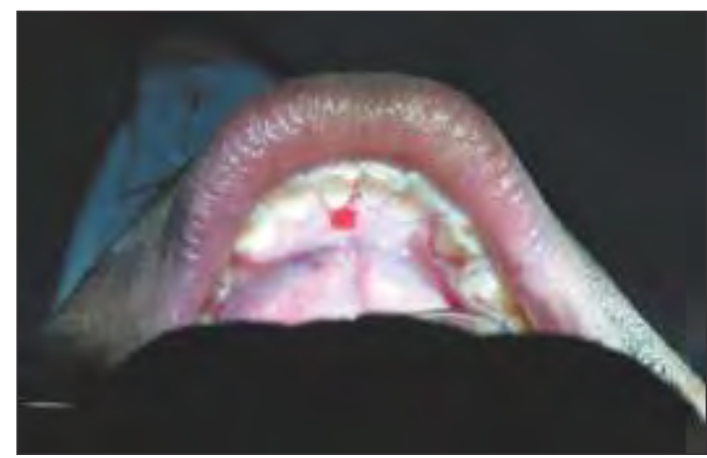

Post-operative

\section{Discussion :}

Pyogenic granuloma, a non-specific conditioned enlargement is a tumour like gingival enlargement that is considered as an exaggerated conditioned response to minor trauma. The lesion varies from a discrete spherical, tumor like mass with a pedunculated attachment to a flattened, keloidlike enlargement with a broad base. It is bright red or purple and either friable to firm, depending on its duration. In majority of cases it presents with ulceration and purulent exudation. The lesion tends to involute spontaneously to become a fibroepithelial papilloma, or may persist relatively unchanged for years. ${ }^{1}$

In 1897, Poncet and Dor first brought the matter to the attention of the surgeons in France under the name of "Botryomycoma hominis". ${ }^{2}$ Hartzell in 1904 gave the term "pyogenic granuloma" or "granuloma pyogenicum". Angelopoulos histologically described it as "hemangiomatous granuloma" due to the presence of numerous blood vessels seen in histological section. Two forms of pyogenic granulomas were described, the lobular capillary hemangioma (LCH) and the non-lobular capillary hemangioma (non-LCH). ${ }^{3}$

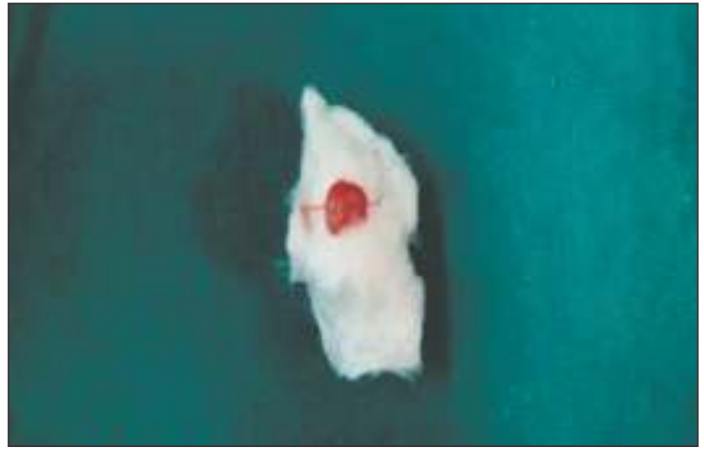

Excised pyogenic granuloma

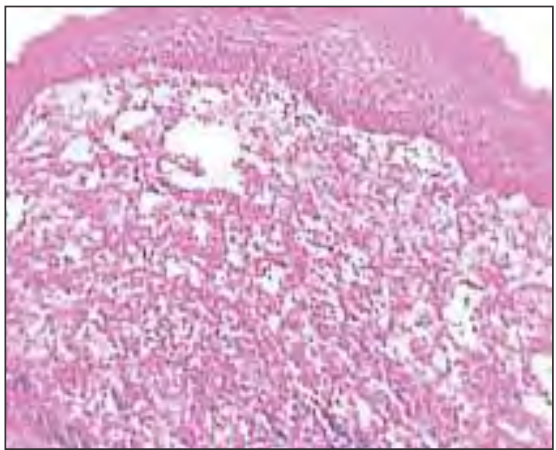

Histological section

Pyogenic granuloma is a commonly occurring inflammatory hyperplasia of the skin and oral mucosa. ${ }^{3}$ Pyogenic granuloma is the most common lesion constituting upto $57 \%$ of all cases of focal reactive gingival lesions. The female: male ratio is $1.7: 1 .{ }^{4}$ Females are more susceptible than males because of the hormonal changes that occur in women during puberty, pregnancy and menopause. ${ }^{5} \mathrm{M}$ ost of the lesions, upto $51.6 \%$ occurred in the incisor/canine region. ${ }^{4}$ Pyogenic granuloma occurs in all ages with a peak incidence of $2^{\text {nd }}$ and $3^{\text {rd }}$ decades of life. ${ }^{6}$ It is not associated with pus as its name suggests and histologically it resembles an angiomatous lesion rather than a granulomatous lesion. It is known by a variety of names such as Crocker and Hartzell's disease, granuloma pyogenicum, granuloma pediculatum benignum, benign vascular tumour and during pregnancy as granuloma gravidarum. This tumour like growth is considered to be non-neoplastic in nature and it presents itself in the oral cavity in various clinical and histological forms. ${ }^{3}$

Various causes like chronic low grade trauma, physical trauma, hormonal factors, bacteria, viruses and certain drugs have been implicated as causative factors in the 
development of pyogenic granulomas. Oral pyogenic granulomas show a predilection for the gingiva, accounting for $75 \%$ of the cases. Local irritants such as calculus, foreign material in the gingiva and poor oral hygiene are the precipitating factors. ${ }^{3}$ Studies have shown that pyogenic granuloma as a hyperplastic, neovascular response to an angiogenic stimulus with imbalance of promotors and inhibitors. Angiogenic growth factors such as vascular endothelial growth factors (VEGF) and decorin, transcription factors (PATF 2 and Pstat3) and signal transduction pathways (MAPK) are overexpressed in pyogenic granulomas. ${ }^{6}$

The result is the exuberant production of granulation tissue producing a dark red or purplish polypoid mass extending from the gingival crevice. The surface is usually smooth but, because of its location, there is normally some surface ulceration with a fibrinous covering. ${ }^{7}$

Radiographic findings are usually absent. However, Angelopoulos concluded that in some cases long standing gingival pyogenic granulomas caused localized alveolar bone resorption. ${ }^{3}$ Excision and biopsy of the lesion is the recommended line of treatment unless it would produce a marked deformity and in such a case incisional biopsy is recommended. Conservative surgical excision of the lesion with removal of irritants such as plaque, calculus and foreign materials is recommended for small painless nonbleeding lesions. Excision of the gingival lesions up to the periosteum with thorough scaling and root planning of adjacent teeth to remove all visible sources of irritation is recommended. Various other treatment modalities such as use of $\mathrm{Nd}$ : YAG laser, carbon dioxide laser, flash lamp

\section{References :}

1. Carranza;s Clinical Periodontology $10^{\text {th }}$ edition page 383

2. Douglass W Montgomery and George D Culver :Granuloma pyogenicum; Arch Derm Syphilol 1932;26(1):131-138.3

3. Sheiba R. Gomes, Quaid Johar Shakir, Prarthana V. Thaker, and Jamshed K. Tavadia: Pyogenic granuloma of the gingiva: A misnomer? - A case report and review of literature ; Journal of Indian Socirty of periodontology 2013Jul-Aug; 17(4): 514-519.

4. O. A.Effiom, W.L. Adeyemo and O.O.Soyele:focal reactive lesions of the gingiva : an analysis of 314 cases at a tertiary health institution in Nigeria;Niger M edJ.2011 Jan-M arch:52(1):35-40. pulse dye laser, cryosurgery, electrodessication, sodium tetradecyl sulfate sclerotherapy and use of intra lesional steroids have been used by various clinicians. The treatment of pyogenic granuloma consists of removal of the lesion with the elimination of irritating local factors. ${ }^{3}$ The recurrence rate is about $15 \% .{ }^{8}$ Taira et al., have shown a recurrence rate of $16 \%$ in excised lesions and also described a case of multiple deep satellite lesions surrounding the original excised lesion in a case of Warner Wilson James syndrome. ${ }^{4}$

Bachymeyer et al and Lee et al reported four cases of oral pyogenic granuloma in chronic graft-verses host disease in patients who were under cyclospoprin. Fowler et al first reported a case in which pyogenic granuloma was found associated with GTR. Differential diagnosis includes peripheral giant cell granuloma, peripheral ossifying fibroma, metastatic cancer, hemangioma, pregnancy tumour, conventional granulation tissue, hyperplastic gingival inflammation, Kaposi's sarcoma, bacillary angiomatosis and Non-Hodgkins lymphoma. ${ }^{9}$

In the case presented here, the patient gave a previous history of recurrence of similar lesion within 4 days of surgical removal. On clinical examination, moderate supragingival and subgingival calculus was detected. Hence the presence of the local factors could be the reason for the lesion to recur. So a thorough scaling and root planning was performed before the surgical excision of the lesion. Satisfactory healing was seen after 1 week of surgery without any sign of recurrence and patient discomfort. There was no recurrence seen after 1 month of follow up.

5. Dr. Sanjay Venugopal, Pyogenic granuloma- A case report J. Dental Research 1:1: pages 80-85

6. Pyogenic granuloma ( lobular capillary hemangioma) Leslie P Lawley.

7. William H Binnie ;periodontal cysts and epulides; periodontology 2000, vol 21,1999 16-32)

8. Carranza;s Clinical Periodontology $11^{\text {th }}$ edition page 126

9. Hamid Jafarzadeh, Majid Sanatkhani, Nooshin Mohtasham; Oral pyogenic granuloma:a review , Journal of oral science, vol no. 4 .167175,2006 . 